\title{
A long period grating directional bend sensor incorporating index modification of the cladding
}

\author{
T. Allsop, M. Dubov, A. Martinez, F. Floreani, I. Khrushchev, D.J. Webb, I. Bennion \\ Authors' Affiliations: \\ T. Allsop, M. Dudov, A. Martinez F. Floreani, I. Khrushchev, D.J. Webb, I. Bennion (Photonics \\ Research Group, Aston University, Aston Triangle, Birmingham, B4 7ET, U.K).
}

\begin{abstract}
Long period gratings written into a standard optical fibre were modified by a femtosecond laser, which produced an asymmetric change to the cladding's refractive index resulting in a directional bend sensor.
\end{abstract}

\section{INTRODUCTION}

A long Period Grating (LPG) is an axially periodic refractive index variation in the core of a single-mode optical fibre that couples light in the core to fibre cladding modes at discrete wavelengths [1]; LPGs have been suggested for many potential applications in the field of sensing [2]. A promising application of fibre LPG devices is as a curvature sensor [3], but a problem here is that in a symmetrical fibre the direction of the bend cannot be determined and thus at present LPGs written in standard fibre cannot be used as true orientation sensors. Some limited success in making orientation sensors has been achieved with LPGs written into asymmetric fibres such as D-shaped cladding fibre [4] or eccentric core fibre [5]. Whilst the resulting devices can determine the orientation of the bend, they still present some practical problems; for example, the cost of the special fibre and the problem of splicing to standard optical fibre. This adds complexity to the fabrication procedure, which raises the cost of the sensor arrays.

In this paper we report for the first time, to our knowledge, the fabrication and characterisation of a LPG sensor in standard telecommunication fibre that can distinguish the direction of bend.

\section{EXPERIMENT AND RESULTS}

The sensing device was fabricated in two stages: the first stage involved LPG inscription and the second the introduction of an asymmetric change in the cladding's refractive index. For the first stage, the LPG was inscribed into a single mode standard communications step-index optical fibre with a core radius of $3.5 \mu \mathrm{m}$ and an outer cladding radius of $62.5 \mu \mathrm{m}$. The fibre was not specifically designed to be photosensitive and so its photosensitivity was increased by hydrogenation at a pressure of 120 Bar for a period of 2 weeks at room temperature. The LPG was fabricated using a frequency doubled argon ion laser with a point-by-point writing technique. The LPGs grating period was $400 \mu \mathrm{m}$ and the length $5 \mathrm{~cm}$.

In the second stage, femtosecond laser radiation was focused at a predetermined point near the core with a $\times 100$ $(\mathrm{NA}=0.55)$ microscopic objective, while the fibre was moved in a direction perpendicular to the laser light and parallel to the fibre core axis. The translation speed was $100 \mu \mathrm{m} / \mathrm{sec}$. A cylindrical lens $(\mathrm{f}=100 \mathrm{~mm})$ was put in the proximity of the microscopic objective to shape the laser beam at the point of inscription. The femtosecond laser system produces a $1 \mathrm{kHz}$ train of $150 \mathrm{fs}$ pulses at $800 \mathrm{~nm}$, and the pulse energy was approximately $1 \mu \mathrm{J}$ after attenuation. Other details of the experimental set-up can be found elsewhere [6].

Two LPG fibre devices (period $400 \mu \mathrm{m}$ and length $5 \mathrm{~cm}$ ) were modified by the femtosecond laser radiation. Firstly, in LPG1 three continuous waveguides (refractive index change of about $1.5 \times 10^{-3}$ ) were written in the same plane and parallel to the core. The intensity of the laser was kept at a level to make single spots overlap, thus making the waveguides continuous. Two of the waveguides were placed $2 \mu \mathrm{m}$ either side of the core and the third waveguide was placed $20 \mu \mathrm{m}$ from the core. The diameter of each waveguide was approximately $5 \mu \mathrm{m}$. Secondly in LPG2 a single continuous waveguide (diameter $\sim 5 \mu \mathrm{m}$ ) was written in the same plane and parallel to the core at a distance of $5 \mu \mathrm{m}$ from the core.

The LPG devices were inserted into a $4 \mathrm{~mm}$ diameter flexible rubber tube and both the fibre and the rubber tube were clamped between two towers with one of the clamps mounted on a translation stage. This was moved inwards to induce a bend in the rubber tube and hence the optical fibre. We describe the bending as convex when the waveguides written $20 \mu \mathrm{m}$ from the core for LPG1 and $5 \mu \mathrm{m}$ from the core for LPG2 are on the outermost side of the bend, and concave when these waveguides are on the inside of the bend. The rubber tube helped ensure that the fibre does not flip to another orientation. For this arrangement, where the LPG is mid-way between the clamps, the sensor's curvature, $R$, is given by [7] 


$$
R=\frac{2 \cdot d}{\left(d^{2}+L^{2}\right)}
$$

where $L$ is the half distance between the edges of the two towers and $d$ is the bending displacement at the centre of the LPG. The experimental characterisation of the LPG's attenuation bands was carried out by illuminating the device using a broadband light source and observing the transmission spectrum with an optical spectrum analyser with an accuracy of $0.05 \mathrm{~nm}$. Examples of the transmission spectra of LPG1 are shown in Figure 1 for concave and convex bending.

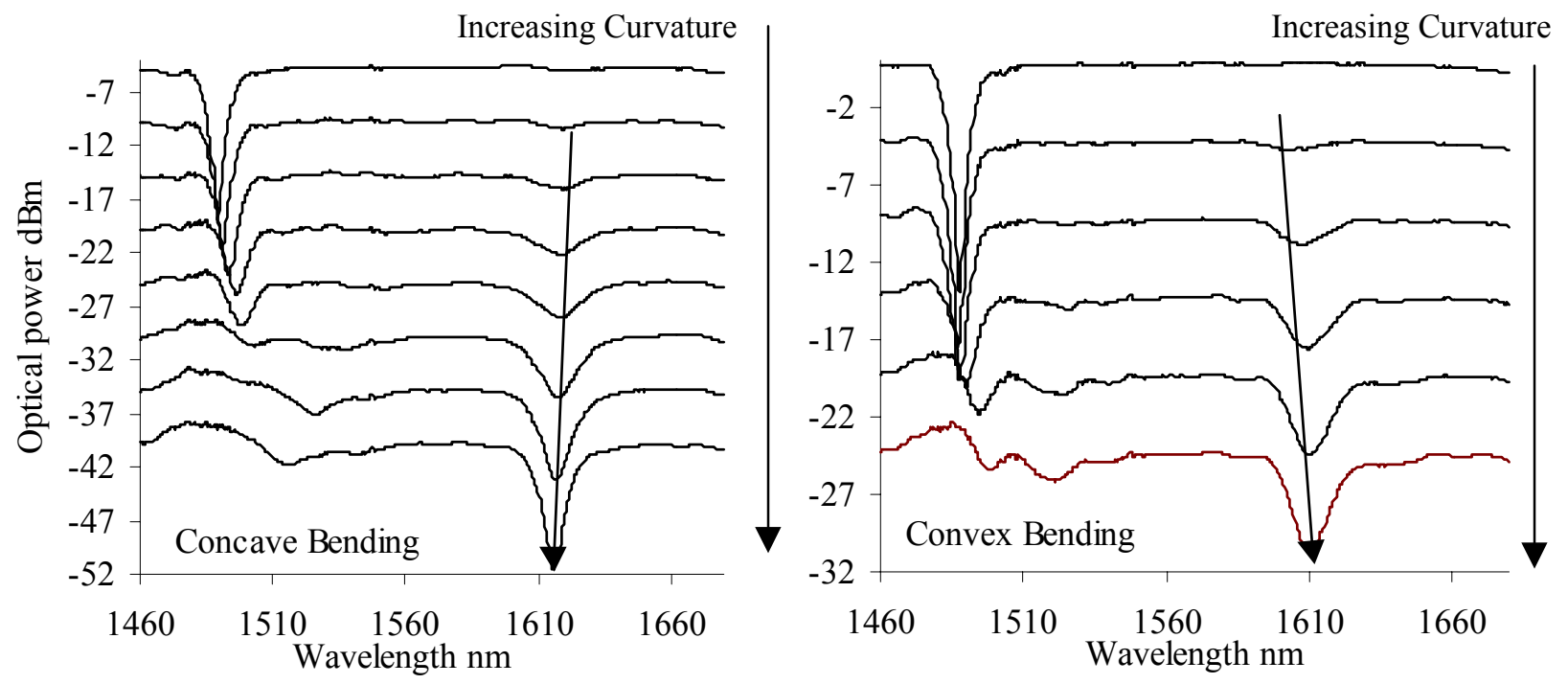

Figure 1. The transmission spectra of LPG1 as a function of direction and amount of curvature.

Firstly, the spectral sensitivity of the normal attenuation bands (those present in the absence of any bending) was measured for both modes of bending, resulting in $\mathrm{d} \lambda / \mathrm{d} R+5.6 \pm 0.1 \mathrm{~nm} \mathrm{~m}$ for concave bending and $\mathrm{d} \lambda / \mathrm{d} R+4.6 \pm 0.2$ $\mathrm{nm} \mathrm{m}$ for convex bending. Secondly the bend induced attenuation band was characterised and displayed a spectral sensitivity of $\mathrm{d} \lambda / \mathrm{d} R-1.6 \pm 0.1 \mathrm{~nm}$ m for concave bending and $\mathrm{d} \lambda / \mathrm{d} R+3.8 \pm 0.5 \mathrm{~nm} \mathrm{~m}$ for convex bending. The wavelength shifts as a function of curvature of the normal and the bend induced attenuation bands of LPG1are shown in figure 2, while figure 3 shows how the asymmetrisation affects the behaviour of the bend-induced attenuation bands in LPG2. For LPG2, whilst the bend-induced attenuation band shows a dramatic change in its spectral response to curvature as a result of the fs inscription, the normal attenuation band close to $1500 \mathrm{~nm}$ shows very little change, and is still unable to distinguish the bend direction.
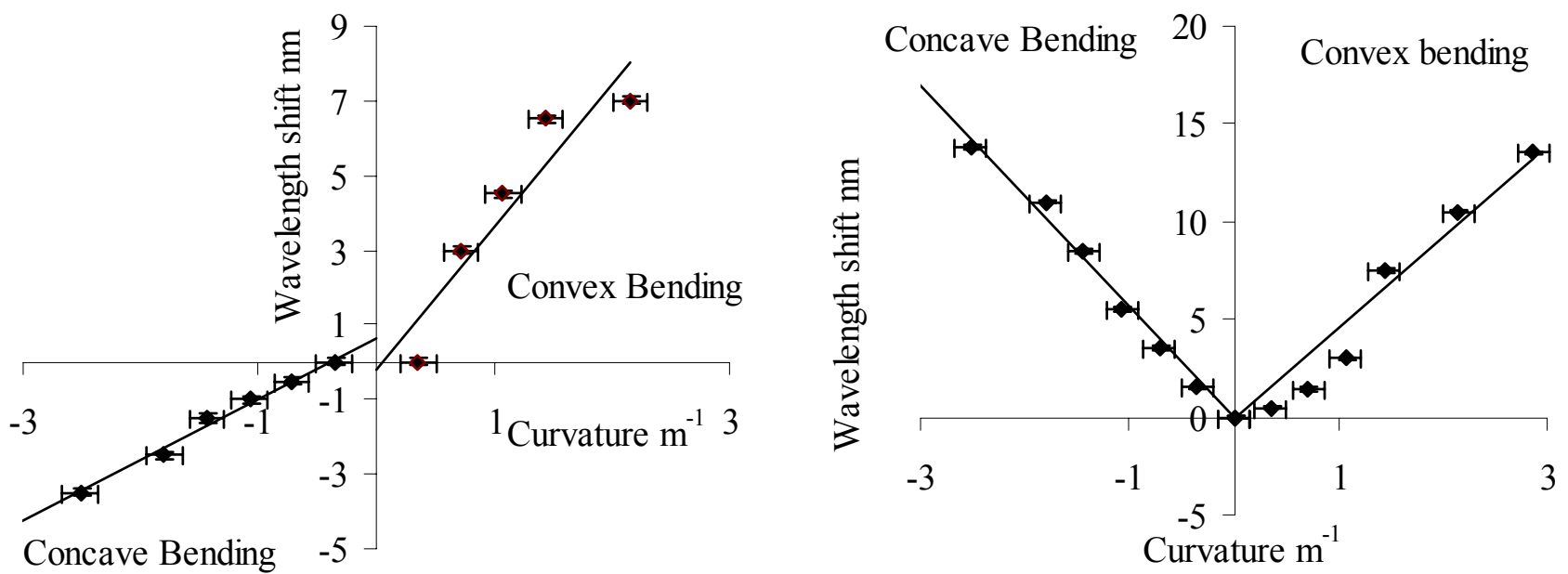

Figure 2. The spectral sensitivity of the induced attenuation bands (left) and the normal attenuation band (right) at $1500 \mathrm{~nm}$ for LPG1. 

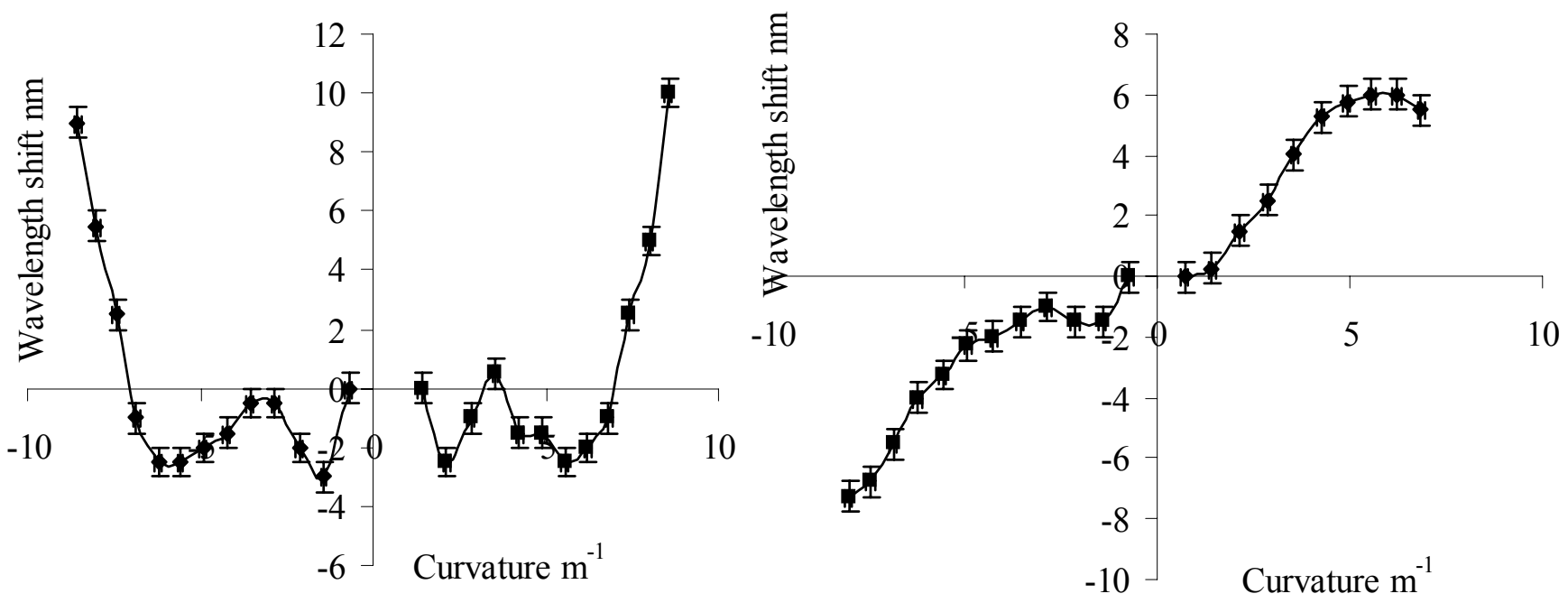

Figure 3. Example of the spectral sensitivity of the induced attenuation band before (left) and after (right) femtosecond laser modification of LPG2.

This spectral behaviour is explained by the introduction of the asymmetry as seen previously in the response to the bending of LPGs in D-shaped fibre [4]. LPG1 was modelled using a finite element package (Femlab) to obtain the effective refractive indices of the core and the cladding modes at various curvatures (concave and convex bending) at a given wavelength. The presence of bending was treated by using the conformal mapping technique [8], which replaces the curved optical fibre waveguide by an equivalent straight waveguide with the following index profile as a function of curvature $R$ and longitudinal strain $\varepsilon$

$$
n(R) \rightarrow\left(n(0, \lambda)+\frac{d n}{d \varepsilon} \cdot \varepsilon\right) \cdot \exp \left(\frac{d}{R}\right)
$$

where $n(0)$ is the initial refractive index of the core/cladding at a wavelength of $\lambda, R$ is the curvature experienced by the fibre and $d$ is the distance from the centre of the fibre. The theoretical wavelength shift was obtained using the same approach as REF 4 and REF 6 giving

$$
\Delta \lambda=\left[\frac{\lambda}{\left(\delta n_{e f f}-\delta n_{g}\right)} \cdot \frac{d \delta n_{\text {eff }}}{d R}+\frac{\left(\delta n_{\text {eff }}\right)^{3}}{\delta n_{g}} \frac{d \Lambda}{d R}\right] \cdot \Delta R
$$

where $\delta n_{\text {eff }}=n_{\text {coeff }}-n_{\text {cleff }_{v}}$ is the differential effective index between the $4^{\text {th }}$ cladding mode and the core mode and $\delta n_{g}=n_{\operatorname{cog}}-n_{\operatorname{clg}_{v}}$ is the differential group index. The effective refractive indices of the core and $4^{\text {th }}$ cladding mode were calculated as a function of curvature from which the wavelength shift of the attenuation band was determined, see figure 4 . 


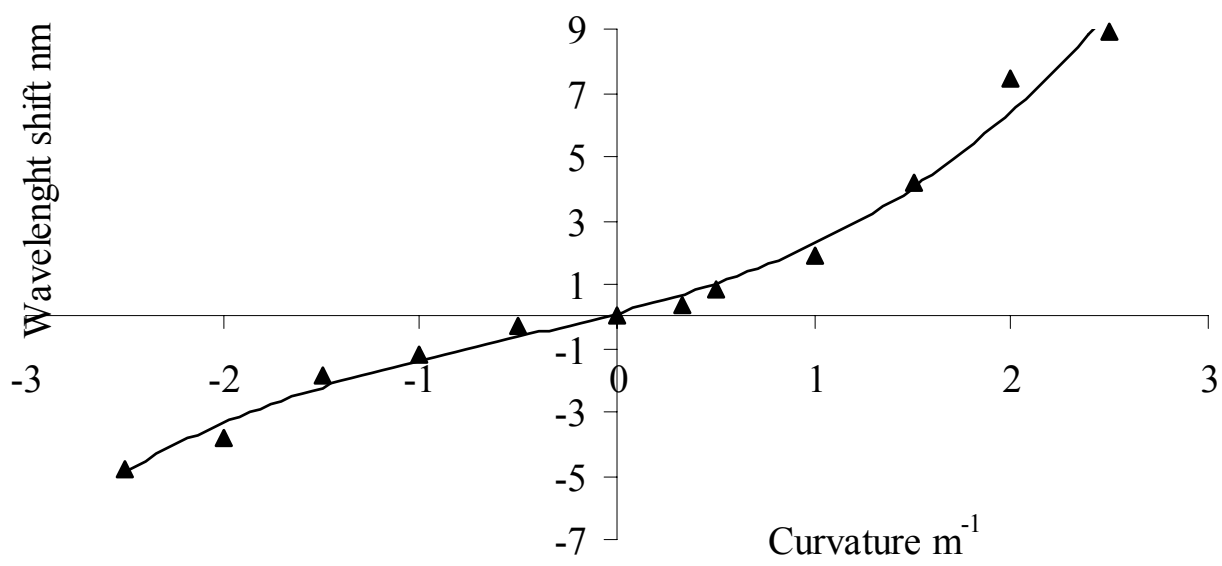

Figure 4. The theoretical spectral behaviour of the LPG fibre device described by the waveguide refractive index geometry of LPG1, showing the $4^{\text {th }}$ cladding mode as a function of curvature.

The modelling produced the same general spectral behaviour with respect to curvature, though the spectral sensitivities of the various attenuation bands were different; this is not surprising since the exact refractive index profile of the LPG device is not known.

\section{CONCLUSION}

We have fabricated a fibre LPG device in standard fibre, which has shown both blue and red wavelength shifts, depending upon the direction of bending experienced by the LPG. This new type of sensor has distinct potential advantages over other orientation-sensitive LPGs in that it only requires standard single mode fibre. This allows simple integration of the sensor into the rest of the system.

\section{REFERENCES}

[1] T. Erdogan, "Cladding-mode resonances in short- and long-period fibre Grating filters", J.Opt.Soc.Am. A, 1997, vol. 4, no.8, pp1760-1773.

[2] A.D. Kersey et al, "Fibre Grating Sensors", J. Lightwave Technol., 1997, vol. 15, no. 8, pp.1442-1462.

[3] T. Allsop et al, "Embedded Progressive-Three Layered fibre long-period gratings for respiratory monitoring", SPIE Biomedical Optics, 2003

[4] T. Allsop, et al, "The Spectral Characteristics of Long Period Gratings written in D-Shaped Optical Fibre as a Bending Sensors", paper TuP-8, OFS2003, Nara Japan, Oct 2003

[5] H.J. Patrick, "Self-aligning, bipolar bend transducer based on long period grating written in eccentric core fibre", Electron. Letts., vol. 36, no. 21, p. 1763-1764, 2000.

[6] A. Martinez, et al, "Point by point FBG inscription by a focused NIR femtosecond laser", OSA Conf. Proc. CLEO/IQEC and PhAST Washington, DC, 2004, CMY6

[7] W. Du, H Tam, M. Liu, X. Tao, "Long-period grating bending sensors in laminated composite structures", SPIE Conf. Proc. Smart structures and materials, San Diego 1998, SPIE Vol 3330, pp284.

[8] M. Heiblum, J.H. Harris, "Analysis of curved optical waveguides by conformal transformation", Quant. Electron., vol.QE-11, no.2, pp. 75-83, 1975. 